\title{
A Prospective Experimental Comparative Study on the Clinical Effects of Calculus Dissolution based Oral Rinse in Gingivitis Patients
}

\author{
Rajiv Saini
}

\section{ABSTRACT}

Aim: The present study was aimed to evaluate the efficacy of calculus/tartar dissolution based oral rinse as an adjunct to toothbrushing in gingivitis patients for long-term effects on new supragingival calculus formation.

Materials and methods: After undergoing a dental prophylaxis, 60 gingivitis subjects with a moderate rate of calculus formation were stratified and randomly assigned to one of two groups: Control group (regular dentifrice alone) and experimental group (using a regular dentifrice and a calculus/tartar control mouthrinse). Subjects brushed and rinsed twice daily, unsupervised, for 6 months. All subjects were assessed with gingival index $(\mathrm{GI})$, plaque index $(\mathrm{PI})$ and calculus levels using the Volpe-Manhold Index (VMI) after 3 and 6 months.

Results: Statistical analysis found that the experimental group (which used a calculus/tartar contain mouthrinse) demonstrated statistically significantly lower VMI scores $(p=0.001$ ) than control group (which used a regular dentifrice alone). In experiment test group $\mathrm{A}$, there was decline in VMI score from 3 to 6 months by $23.12 \%$ as compared to group $B$ where there was continuous increase in VMI scores from 3 to 6 months by $22.16 \%$.

Conclusion: A mouthrinse containing tetrapotassium pyrophosphate and sodium tripolyphosphate as the anticalculus agent provides a clinically relevant reduction in calculus formation in subjects with a moderate rate of such formation.

Keywords: Anticalculus, Antitartar, Mouthrinse, Calculus.

How to cite this article: Saini R. A Prospective Experimental Comparative Study on the Clinical Effects of Calculus Dissolution based Oral Rinse in Gingivitis Patients. Int J Experiment Dent Sci 2015;4(1):33-39.

Source of support: Nil

Conflict of interest: None

\section{Associate Professor}

Department of Periodontology and Oral Implantology, Pravara Institute of Medical Sciences, Ahmednagar, Maharashtra India

Corresponding Author: Rajiv Saini, Associate Professor Department of Periodontology and Oral Implantology, Pravara Institute of Medical Sciences, Loni, Ahmednagar, Maharashtra India, e-mail: drperiodontist@yahoo.co.in

\section{INTRODUCTION}

The microorganisms found in the human oral cavity have been referred to as the oral microflora, oral microbiota, or more recently as the oral microbiome. ${ }^{1}$ Approximately, 280 bacterial species from the oral cavity have been isolated in culture and formally named. It has been estimated that less than half of the bacterial species present in the oral cavity can be cultivated using anaerobic microbiological methods and that there are likely 500 to 700 common oral species. Cultivation-independent molecular methods, primarily using $16 \mathrm{~S}$ rRNA genebased cloning studies, have validated these estimates by identifying approximately 600 species or phylotypes. ${ }^{2}$ It is estimated over $95 \%$ of bacteria existing in nature are in biofilms. ${ }^{3}$ Biofilms are ubiquitous; they form on virtually all surfaces immersed in natural aqueous environments. Biofilms form particularly fast in flow systems where a regular nutrient supply is provided to the bacteria. The reason for the existence of the biofilm is that it allows the microorganisms to stick and to multiply on surfaces. ${ }^{4}$

Periodontal diseases, including gingivitis and periodontitis, are among the most common chronic diseases and studies in the USA and the UK suggest that some degree of gingivitis affects 50 to $90 \%$ of the adult population. ${ }^{5}$ Gingivitis, or inflammation of the gums, occurs in response to the bacteria in plaque that accumulates near the gum line. It is characterized by redness, swelling or bleeding of the gums. Periodontitis is inflammation of the tissues surrounding the tooth affecting the gingiva, periodontal ligaments and the bone and in its severe forms there can be loss of bone that supports the tooth, resulting in the tooth becoming loose and even causing tooth loss. The loss of supporting structures can result in the formation of 'pockets' between the gum and the tooth. Gingivitis is the initial stage of disease progression and can further extend to periodontitis if the bacterial bio burden is not eradicated by the optimal means.

Many patients with gingivitis have calculus or other associated local factors, e.g. defective dental restorations that interfere with personal oral hygiene and the ability to remove bacterial plaque. An acceptable therapeutic result 
for these individuals is usually obtained when personal plaque control measures are performed in conjunction with professional removal of plaque, calculus and other local contributing factors. ${ }^{6,7}$

Dental plaque biofilm cannot be eliminated. However, the pathogenic nature of the dental plaque biofilm can be reduced by reducing the bio-burden (total microbial load and different pathogenic isolates within that dental plaque biofilm) and maintaining a normal flora with appropriate oral hygiene methods. ${ }^{8}$ Such control is not generally achieved by mechanical oral hygiene procedures alone. Thus, there is a clear rationale for the use of chemical anti-plaque agents to augment mechanical means. The principle routes to chemical plaque control are to prevent colonization of the tooth surface, to inhibit the growth of microorganisms, the prevent plaque maturation, to modify plaque biochemistry and to modify plaque ecology to a less pathogenic flora. Several alcohol based anti-plaque agents like are available in the market and most commonly chlorhexidine gluconate (CHX); but with scientifically proven side-effects associated with CHX, i.e. temporary loss of taste; staining of teeth, restorations, and mucosa; dryness and soreness of mucosa; bitter taste; and slight increase in supragingival calculus formation ${ }^{9,10}$ it cannot be advocated for longer duration of time. Also, most of the oral rinses are based on antimicrobial properties and no emphasis on the dissolution of dental calculus, which actually serves as a reservoir to dental plaque. In recent years, there has been focus on therapeutics agents that will focus more on eradication for calculus from the gum line.

Dental calculus or tartar is an adherent calcified mass that form on the surface of teeth and dental appliance through mineralization of bacterial dental plaque in aqueous environment. Dental calculus is layered by nonmineralized bacterial plaque; hence, act as reservoir of microorganisms in the oral cavity. Various studies carried out to reveal the presence of calculus have shown that calculus is present in 70 to $100 \%$ cases. These studies do not discriminate between supra and subgingival calculus but they indicate high prevalence of calculus in all studied populations. ${ }^{11}$
Hence, this study was aimed to evaluate the efficacy of calculus dissolution based oral rinse as an adjunct to toothbrushing in gingivitis patients for long-term effects on new supragingival calculus formation.

\section{MATERIALS AND METHODS}

The present clinic study was conducted in the department of periodontology, after approved from the research and ethical committee of Pravara Institute of Medical Sciences, Loni, Ahmednagar, Maharashtra, India.

\section{Patient Selection}

The subjects enrolled in this study were selected from the out patient department (OPD) of Periodontology, Dental College and Hospital, Loni, Maharashtra, India. It was a randomized, double blinded clinical study. After an informed consent, a total 60 gingivitis patients between the ages of 18 and 55 years were enrolled in the study and divided under two categories. Each group was comprised of 30 subjects each as illustrated in Table 1.

\section{Criteria for Patient Selection}

\section{Inclusion Criteria}

(1) Age-group between 18 and 55, (2) willing to participate in the study, (3) the patient should have a minimum of 20 sound permanent teeth with minimum of 5 teeth to be present in each arch quadrant, (4) presence of all lower arch (mandible) anterior teeth, (5) subjects with moderate to severe gingivitis, i.e. gingival index score between 2 and 3, (6) mean volpe manhold calculus index (VMI) score of at least $7 \mathrm{~mm}$ on lingual surfaces of the 6 mandibular anterior teeth, (7) systemically healthy subjects.

\section{Exclusion Criteria}

(1) Presence of any neurological disorder, (2) presence of a disease with possible effects on the immune system (e.g. chronic infections or cancer), (3) patient who have received antibiotics or NSAIDS (like Ibuprofen) in past 9 to 11 weeks and other medicines which could alter the oral microbial flora were excluded, (4) patients who have received periodontal treatment in past 6 months,

Table 1: Age and sex wise distribution in groups $A$ and $B$

\begin{tabular}{|c|c|c|c|c|c|c|}
\hline \multirow[b]{2}{*}{ Age in years } & \multicolumn{3}{|c|}{ Group A (test group) $(n=30)$} & \multicolumn{3}{|c|}{ Group B (control group) $(n=30)$} \\
\hline & Male & Female & Total & Male & Female & Total \\
\hline $20-30$ & 3 & 4 & $7(23.33 \%)$ & 2 & 0 & $2(6.67 \%)$ \\
\hline $30-40$ & 9 & 6 & $15(50 \%)$ & 9 & 8 & $17(56.66 \%)$ \\
\hline $40-50$ & 3 & 4 & $7(23.33 \%)$ & 3 & 5 & $8(26.67 \%)$ \\
\hline $50-60$ & 1 & 0 & $1(3.34 \%)$ & 3 & 0 & $3(10 \%)$ \\
\hline Total & $16(53.33 \%)$ & $14(46.67 \%)$ & 30 & $17(56.66 \%)$ & $13(43.34 \%)$ & 30 \\
\hline Mean \pm SD & $36.06 \pm 9.87$ & $36.57 \pm 8.24$ & $36.30 \pm 7.45$ & $38.15 \pm 10.25$ & $38.53 \pm 9.54$ & $39.23 \pm 8.77$ \\
\hline
\end{tabular}


(5) pregnant and lactating mother, (6) patient with artificial prosthesis, (7) patients who smokes or consumes tobacco in any form, (8) patients suffering with diabetes, arthritis any type of heart disease (MI, CHD, etc.), (9) female patient using intrauterine birth control devices or birth control pills, (10) obese individuals (30 and above range as per WHO BMI cut-off for weight categories for Asians).

\section{Clinical Parameters Recorded}

Clinical parameters that were evaluated were gingival index (GI), plaque index (PI) and Volpe-manhold calculus index (VMI).

\section{Gingival Index}

The gingival index was used in order to assess the severity of gingivitis. The tissues surrounding each tooth were divided into four gingival scoring units: distofacial papilla, facial gingival margin, mesiofacial papilla and the entire lingual gingival margin were examined systematically for each tooth and a score ranging from 0 to 3 was given for each surface. A blunt instrument, such as a periodontal probe was used to assess the bleeding tendency of the tissues. Totalling the score around each tooth and dividing by four yielded the individual score per tooth. Totalling all the scores per tooth and dividing by the number of teeth examined provided the gingival index per person.

\section{Plaque Index}

Plaque was assessed on the distal, facial, mesial, lingual or palatal area of each tooth. These areas were assigned a score between 0 and 3. Plaque score for a tooth was obtained by totalling the score for each area and dividing by four. Plaque score per person was obtained by adding plaque score for each tooth and dividing by the total number of teeth examined.

\section{Volpe-Manhold Calculus Index}

The Volpe-Manhold probe method of calculus assessment was to assess the presence and severity of calculus formation, especially new deposits of supragingival calculus, following an oral prophylaxis. To obtain the VMI scores, the three tooth planes, the mesial, distal, and gingival, on the lingual surface of lower six anterior teeth (i.e. central, lateral and cuspids) are examined. The periodontal probe is used to measure the linear extent of the supragingival calculus by placing the flat calibrated end of the probe always at the most inferior visible border of the calculus formation. In cases where the gum line is unhealthy and displaced, the probe is used to depress the tissue to measure from the inferior border of visible calculus. The calculus is measured in increment of $0.5 \mathrm{~mm}$, from 0 to $5.0 \mathrm{~mm}$. Calculation of VMI, to calculate the VMI score per tooth, the scores for the three planes, the mesial, distal and gingival are summed. Then, all the tooth scores are summed for subject's total VMI score.

\section{Clinical Protocol}

Patients received a verbal description about the clinical protocol to be followed in this clinical trial. Sixty subjects having a PI and GI score between 2 and 3; and mean VMI score of at least $7 \mathrm{~mm}$ on lingual surfaces of the 6 mandibular anterior teeth were selected for this study. The subjects selected for the study were educated regarding the study being carried out and informed consent was obtained. To standardize the procedure and control extraneous variables, each subject was treated by same operator, ultrasonic unit and sterile ultrasonic insert and in the same operatory. In order to have the unbiased and accurate clinical data, we followed a double blind protocol in the study for enrolment of the patients in terms of treatment plan (oral prophylaxis). Also categorizations of patients were done randomly, with oral products regime be followed after the oral prophylaxis. Subjects were then advised to brush twice daily 5 minutes by use of regular dentifrice (without anti-calculus ingredients) with modified bass method technique (technique demonstrated to each subject). Similar dentifrice and medium bristle toothbrushes were provided to each of the subject during the study course to maintain standardization. The subjects then categorized 30 each into two treatment regime in groups A and B as illustrated in Table 2.

\section{Evaluation Protocol}

Baseline clinical measurements were recorded for both the groups. Oral prophylaxis was carried out in patients

Table 2: Subjects grouped under treatment regime

\begin{tabular}{ll}
\hline Groups & Clinical protocol \\
\hline A & $\begin{array}{l}\text { Toothbrushing with regular dentifrice and calculus } \\
\text { dissolution based oral rinse twice daily, i.e. Periogen } \\
\text { with directions to use. Subjects were advised to } \\
\text { dissolve } 1 \text { spoon full of Periogen powder in } 100 \mathrm{ml} \\
\text { of warm water. Swish solution in mouth for at least } \\
1 \text { minute in three 20-second intervals essentially } \\
\text { using all the mixture. Cups with standardized volume } \\
\text { quantity of } 100 \mathrm{ml} \text { were also provided to each subject/ } \\
\text { participant for their compliance. } \\
\text { Toothbrushing with regular dentifrice and not } \\
\text { assigned with application/use of calculus dissolution } \\
\text { oral rinse. }\end{array}$ \\
\hline
\end{tabular}


belonging to both the groups, i.e. A and B. Recall visits was scheduled for all the subjects belonging to both groups (A and B) on 3rd and 6th month and all clinical measurements were recorded.

\section{STATISTICAL ANALYSES}

For statistical analyses, individual measurements were summarized within each individual and then analyzed. Statistical analysis was performed by applying mean, standard deviation (SD), Student's unpaired t-test, Probability (P), analysis of variance (ANOVA), and TukeyKramer multiple-comparison tests and Friedman tests.

\section{RESULTS}

Distributions of mean and standard deviation values of all the clinical parameters of both the groups (A and B) were illustrated in Tables 3 and 4 .

\section{Group A}

By applying Friedman's test (nonparametric repeated measures ANOVA) variation among median values of all clinical parameters were significantly higher than expected by chance in group A compared together $(\mathrm{p}<$ 0.0001), Where value of $\mathrm{Fr}=183.47, \mathrm{p}<0.0001$. By applying Kruskal-Wallis test variation among mean values of all clinical parameters are significantly higher than expected by chance in group A compared together $(\mathrm{p}<$ 0.0001 ). Where value of $\mathrm{F}=955.68, \mathrm{p}<0.0001$, very highly significant. By applying student's paired t-test, there was a highly significant decrease in mean values of all clinical parameters from baseline to 3 months ( $p<0.001$, baseline to 6 months $(\mathrm{p}<0.001)$ and from 3 to 6 months $(\mathrm{p}<0.001)$ in group A as illustrated in Table 5.

The above interpretation scientifically established that oral calculus dissolution based oral rinse, i.e. Periogen along with conventional oral hygiene plays a synergistic role in maintain the long-term oral hygiene by significantly reducing the plaque accumulation and suppressing the gingival inflammation. The results also highlighted that oral rinse used in the study had a notable efficiency in reducing the new calculus formation after oral prophylaxis.

\section{Group B}

By applying Friedman's test (nonparametric repeated measures ANOVA) variation among median values of all clinical parameters were significantly higher than expected by chance in group B compared together $(\mathrm{p}<0.0001)$, where value of $\mathrm{Fr}=232.83, \mathrm{p}<0.0001$. By applying Kruskal-Wallis test variation among mean values of all clinical parameters are significantly higher than expected by chance in group B compared together
Table 3: Distribution of mean and standard deviation (SD) values of clinical parameters in group $A$

\begin{tabular}{llll}
\hline \multirow{2}{*}{$\begin{array}{l}\text { Clinical } \\
\text { parameters }\end{array}$} & \multicolumn{3}{c}{ Group A (test group) $(n=30)$} \\
\cline { 2 - 4 } & Maseline & 3 months & 6 months \\
\hline GI & $2.63 \pm 0.33$ & $0.80 \pm 0.14$ & $0.68 \pm 0.11$ \\
PI & $2.57 \pm 0.31$ & $0.74 \pm 0.13$ & $0.61 \pm 0.09$ \\
VMI & $10.60 \pm 0.82$ & $2.13 \pm 0.99$ & $1.73 \pm 1.15$ \\
\hline
\end{tabular}

GI: Gingival index; PI: Plaque index; VMI: Volpe-Manhold index

Table 4: Distribution of mean and standard deviation (SD) values of clinical parameters in group $B$

\begin{tabular}{llll}
\hline \multirow{2}{*}{$\begin{array}{l}\text { Clinical } \\
\text { parameters }\end{array}$} & \multicolumn{3}{c}{ Group B (control group) $(n=30)$} \\
\cline { 2 - 4 } & Baseline & 3 months & 6 months \\
\cline { 2 - 4 } GI & $2.59 \pm 0.29$ & Mean $\pm S D$ & Mean $\pm S D$ \\
$\mathrm{PI}$ & $2.54 \pm 0.24$ & $0.99 \pm 0.14$ & $1.21 \pm 0.10$ \\
VMI & $10.50 \pm 0.59$ & $4.67 \pm 0.56$ & $5.70 \pm 0.67$ \\
\hline
\end{tabular}

GI: Gingival index; PI: Plaque index; VMI: Volpe-Manhold index

Table 5: Comparative assessment of clinical parameters within group A

\begin{tabular}{llll}
\hline $\begin{array}{l}\text { Clinical } \\
\text { parameters }\end{array}$ & $\begin{array}{l}\text { Baseline to } \\
\text { 3 months }\end{array}$ & $\begin{array}{l}\text { Baseline to } \\
6 \text { months }\end{array}$ & 3 to 6 months \\
\hline GI & $\mathrm{t}=27.02$, & $\mathrm{t}=30.76$, & $\mathrm{t}=3.33$, \\
& $\mathrm{p}<0.001, \mathrm{HS}$ & $\mathrm{p}<0.001, \mathrm{HS}$ & $\mathrm{p}<0.001, \mathrm{HS}$ \\
$\mathrm{PI}$ & $\mathrm{t}=28.86$, & $\mathrm{t}=34.01$, & $\mathrm{t}=4.10$, \\
& $\mathrm{p}<0.001, \mathrm{HS}$ & $\mathrm{p}<0.001, \mathrm{HS}$ & $\mathrm{p}<0.001, \mathrm{HS}$ \\
VMI & $\mathrm{t}=51.19$, & $\mathrm{t}=54.26$, & $\mathrm{t}=3.64$, \\
& $\mathrm{p}<0.001, \mathrm{HS}$ & $\mathrm{p}<0.001, \mathrm{HS}$ & $\mathrm{p}<0.001, \mathrm{HS}$
\end{tabular}

GI: Gingival index; PI: Plaque index; VMI: Volpe-Manhold index; HS: Highly significant; t: t-value; $p$ : $p$-value

$(p<0.0001)$. Where value of $F=2032.7, p<0.0001$, very highly significant. By applying student's paired t-test there is a highly significant decrease in mean values of all clinical parameters from baseline to 3 months ( $p<$ 0.001 , baseline to 6 months $(\mathrm{p}<0.001)$ and from 3 to 6 months ( $\mathrm{p}<0.001)$ in group B as illustrated in Table 6.

The above interpretation scientifically established that conventional oral hygiene plays a effective role in maintain the oral hygiene by significantly reducing the plaque accumulation and suppressing the gingival inflammation for shorter period of time as observed from baseline to 3 months. The results highlighted that

Table 6: Comparative assessment of clinical parameters within group B

\begin{tabular}{llll}
\hline $\begin{array}{l}\text { Clinical } \\
\text { parameters }\end{array}$ & $\begin{array}{l}\text { Baseline to } \\
\text { 3 months }\end{array}$ & $\begin{array}{l}\text { Baseline to } \\
6 \text { months }\end{array}$ & 3 to 6 months \\
\hline GI & $\mathrm{t}=34.19$, & $\mathrm{t}=36.17$, & $\mathrm{t}=11.44$, \\
& $\mathrm{p}<0.001, \mathrm{HS}$ & $\mathrm{p}<0.001, \mathrm{HS}$ & $\mathrm{p}<0.001, \mathrm{HS}$ \\
$\mathrm{PI}$ & $\mathrm{t}=44.70$, & $\mathrm{t}=42.87$, & $\mathrm{t}=10.05$, \\
& $\mathrm{p}<0.001, \mathrm{HS}$ & $\mathrm{p}<0.001, \mathrm{HS}$ & $\mathrm{p}<0.001, \mathrm{HS}$ \\
VMI & $\mathrm{t}=55.56$, & $\mathrm{t}=41.75$, & $\mathrm{t}=9.17$, \\
& $\mathrm{p}<0.001, \mathrm{HS}$ & $\mathrm{p}<0.001, \mathrm{HS}$ & $\mathrm{p}<0.001, \mathrm{HS}$ \\
\hline
\end{tabular}

GI: Gingival index; PI: Plaque index; VMI: Volpe-Manhold index; HS: Highly significant; t: t-value; $p$ : p-value 
A Prospective Experimental Comparative Study on the Clinical Effects of Calculus Dissolution based Oral Rinse

Table 7: Comparison of mean and SD values of clinical parameters in groups A and B at baseline, 3 and 6 months

\begin{tabular}{|c|c|c|c|c|c|c|}
\hline \multirow[b]{3}{*}{ Clinical parameters } & \multicolumn{3}{|c|}{ Group A (test group) $(n=30)$} & \multicolumn{3}{|c|}{ Group B (control group) $(n=30)$} \\
\hline & Baseline & 3 months & 6 months & Baseline & 3 months & 6 months \\
\hline & Mean $\pm S D$ & Mean $\pm S D$ & Mean $\pm S D$ & Mean $\pm S D$ & Mean $\pm S D$ & Mean $\pm S D$ \\
\hline $\mathrm{GI}$ & $2.63 \pm 0.33$ & $0.80 \pm 0.14$ & $0.68 \pm 0.11$ & $2.59 \pm 0.29$ & $1.03 \pm 0.12$ & $1.27 \pm 0.11$ \\
\hline $\mathrm{PI}$ & $2.57 \pm 0.31$ & $0.74 \pm 0.13$ & $0.61 \pm 0.09$ & $2.54 \pm 0.24$ & $0.99 \pm 0.14$ & $1.21 \pm 0.10$ \\
\hline VMI & $10.60 \pm 0.82$ & $2.13 \pm 0.99$ & $1.73 \pm 1.15$ & $10.50 \pm 0.59$ & $4.67 \pm 0.56$ & $5.70 \pm 0.67$ \\
\hline
\end{tabular}

GI: Gingival index; PI: Plaque index; VMI: Volpe-Manhold index

Table 8: Comparative assessment of clinical parameters of group A with B

\begin{tabular}{lll}
\hline Clinical & \multicolumn{2}{c}{ Groups A vs B } \\
\cline { 2 - 3 } parameters & From 3 month & From 6 month \\
\hline GI & $\mathrm{t}=10.50, \mathrm{p}<0.001, \mathrm{HS}$ & $\mathrm{t}=11.07, \mathrm{p}<0.001, \mathrm{HS}$ \\
$\mathrm{PI}$ & $\mathrm{t}=8.33, \mathrm{p}<0.001, \mathrm{HS} \quad \mathrm{t}=13.33, \mathrm{p}<0.001, \mathrm{HS}$ \\
VMI & $\mathrm{t}=12.27, \mathrm{p}<0.001, \mathrm{HS} \quad \mathrm{t}=16.34, \mathrm{p}<0.001, \mathrm{HS}$ \\
\hline
\end{tabular}

GI: Gingival index; PI: plaque index; VMI: Volpe-Manhold index; HS: highly significant; $t$ : t-value; $p$ : $p$-value

long-term advantages of only conventional oral hygiene were limited and there was significantly increase over all the clinical parameters from 3 to 6 months including new calculus formation.

\section{Group A vs Group B}

Comparisons of mean and standard deviation values of all the clinical parameters of both the groups (A and B) were illustrated in Table 7. By applying Kruskal-Wallis test, variation among mean values of all clinical parameters are significantly higher than expected by chance in groups A and B compared together, where value of $F=1249.87$, $\mathrm{p}<0.0001$, very highly significant. By applying Student's unpaired t-test, there was a highly significant difference in mean values of GI, PI and VMI, from 3 to 6 months ( $\mathrm{p}<0.001)$, when compared group A with group B as illustrated in Table 8.

\section{DISCUSSION}

The design of the study has been illustrated in Flow Chart 1. Long-term oral hygiene including prevention of new calculus formation is always a key target for maintaining optimal periodontal health. Professional bi-annually oral prophylaxis plays a vital role in reducing the oral bio burden by removing plaque, calculus and stains from the oral cavity. However, maintaining the home dental oral hygiene especially between 6 months professional oral prophylaxis is very critical. There was lot of research support for traditional alcohol based oral rinse in terms of plaque control but no significant data supporting its effectiveness on anticalculus effects. In this 6 months clinical study, the test product, i.e. Periogen with key ingredient of tetrapotassium pyrophosphate and sodium tripolyphosphate, clinically proved to be a potent oral

\section{Flow Chart 1: Study flow chart}

Seventy-three patients were assessed for eligibility

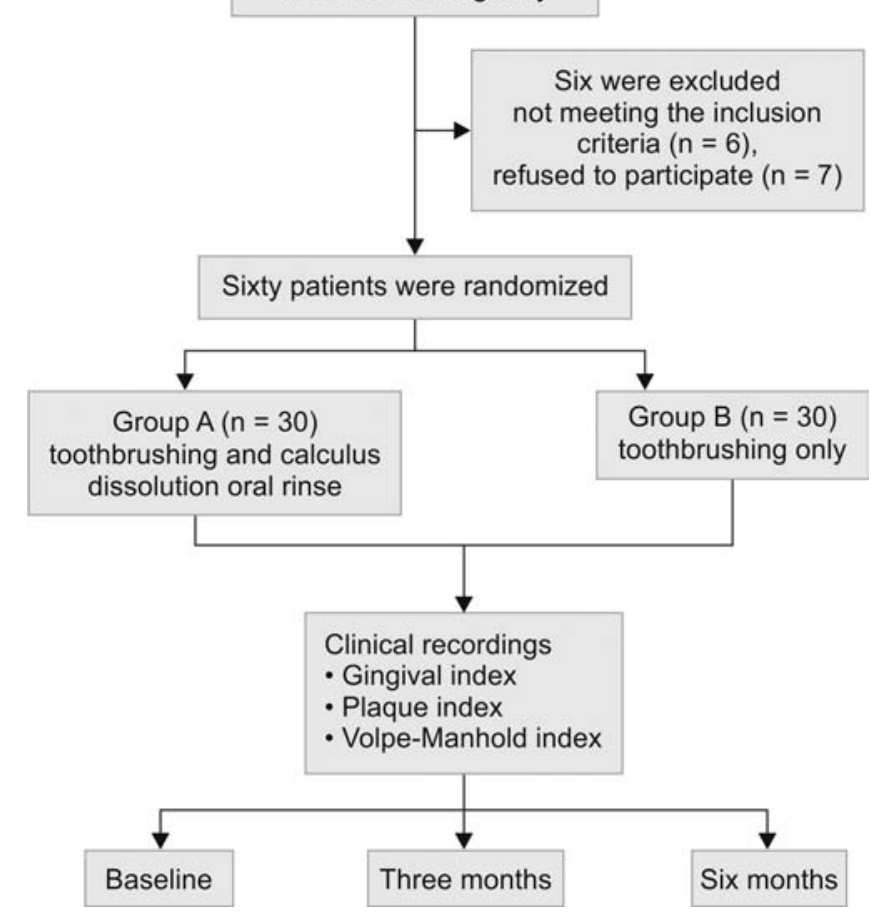

rinse for suppressing the new plaque formation, gingival inflammation and new supragingival calculus formation without any noticeable side effects.

The results of this study showed that there was significant reduction in plaque index and GI in group A (test group) when compared with group B with both long- and short-term clinical recordings. There was 17.64 and $21.31 \%$ reduction of GI and plaque index in group A from 3 to 6 months of clinical recordings. In contrast to that there was 23.39 and 22.64\% increase of GI and PI in Group B from 3 to 6 months of clinical recordings.

The main goal of this study was to evaluate the level of new calculus formation after professional oral prophylaxis in both the groups. In this study, we observed that test group A using Periogen showed a significantly lower amount of new calculus formation in both shortand long-term usage. In this study before evaluation the two groups for their effectiveness of new calculus formation after oral prophylaxis; subjects were chosen that showed equal efficiency of calculus formation and both the groups have a mean VMI of $10.60 \pm 0.82$ and 


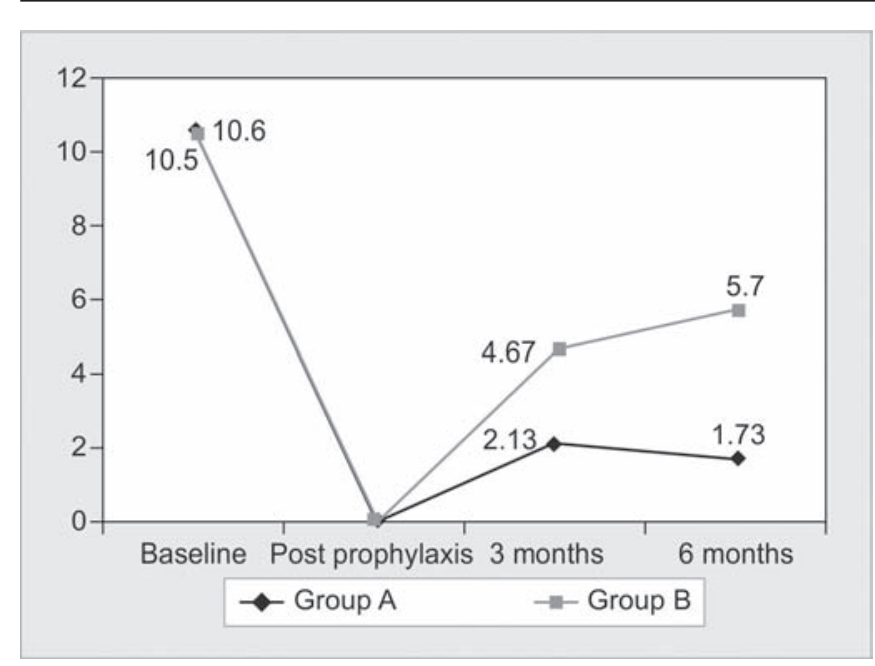

Graph 1: Distribution of mean values Volpe-Manhold index (VMI) in groups $\mathrm{A}$ and $\mathrm{B}$ at baseline, 3 and 6 months

$10.50 \pm 0.59$ respectively. After professional prophylaxis subjects under both the groups were put down with almost no calculus in the oral cavity with VMI score $0 \pm 0$. After 3 months of clinical evaluation, the mean \pm SD value of VMI in the study in groups $\mathrm{A}$ and $\mathrm{B}$ at 3 months was $2.13 \pm 0.99$ and $4.67 \pm 0.56$ respectively. This highly significant difference in VMI scores in both the groups after 12 weeks of time showed benefits of test product over the conventional oral hygiene.

After 6 months of clinical evaluation, the mean \pm $\mathrm{SD}$ value of VMI in the study in groups $\mathrm{A}$ and $\mathrm{B}$ at 3 months was $1.73 \pm 1.15$ and $5.70 \pm 0.67$ respectively. In test group $\mathrm{A}$, there was still decline in VMI score from 3 to 6 months by $23.12 \%$ as compared to group B where there was continuous increase in VMI scores from 3 to 6 months by 22.16\% (Graph 1). The possible mechanism for the continuous decrease in the new calculus formation in test group A is due to regular use of anticalculus oral rinse (Periogen) used in this study along with regular oral hygiene regime. Anticalculus oral rinse (Periogen) is comprised of five innovative key ingredients: tetrapotassium pyrophosphate, sodium tripolyphosphate, baking soda, citric acid and a small amount of fluoride. When added to water, ingredients baking soda and citric acid add fizz in order to draw the active tartar-dissolving ingredients into solution and to establish a healthy $7.6 \mathrm{pH}$.

Tetrapotassium pyrophosphate is a colorless transparent crystalline chemical compound with the formula $\mathrm{Na}_{4} \mathrm{P}_{2} \mathrm{O}_{7}$. It is a salt composed of pyrophosphate and
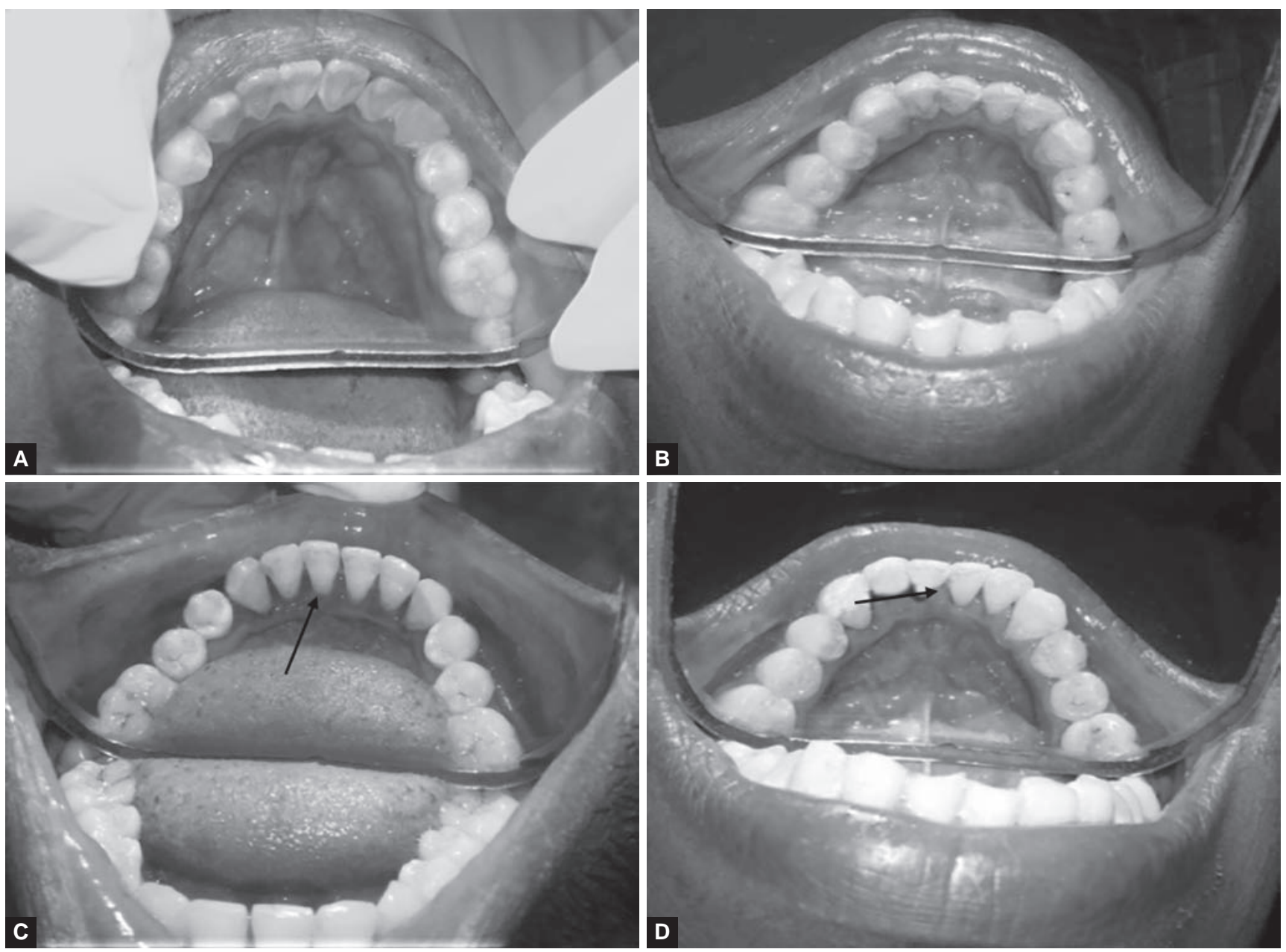

Figs 1A to D: Clinical pictures of reduced calculus deposits under group A: (A) Case 1-before,

(B) case 2-before, (C) case 1-after and (D) case 2-after 
sodium ions. It is the 'tartar control' agent which removes calcium and magnesium from the saliva, so they cannot deposit on the teeth. It has been shown that pyrophosphates has a high affinity to hydroxyapatite (HA) surfaces, probably by an interaction with $\mathrm{Ca}^{2+}$ in the hydration layer. By interacting with HA and enamel surface, pyrophosphate reduces their protein binding capacity. It also has the ability to inhibit calcium phosphate formation. It is, therefore, conceivable that pyrophosphates introduce in the oral cavity through dentifrices may affect pellicle formation. ${ }^{12}$ Tetrapotassium pyrophosphate inhibits the mineralization of biofilm before it is transferred into supragingival calculus. ${ }^{13}$ Sodium tripolyphosphate is an inorganic compound with formula $\mathrm{Na}_{5} \mathrm{P}_{3} \mathrm{O}_{10}$. It is a mineral based ingredient that binds the calcium present in saliva and reduces the formation of tartar on the tooth surface.

This clinical study showed that the presence of tetrapotassium pyrophosphate and sodium tripolyphosphate in mouthwash solution significantly inhibited the development of dental calculus. Thus, the tetrapotassium pyrophosphate and sodium tripolyphosphate treatment formulated with patented balance mixture resulted in a reduction of tartar formation as a result of reduced calcification of dental plaque (Figs 1A to D).

In addition, when questioned, none of the subjects reported any experience of adverse effects, related or non-related to the study products, at any of the clinical visits. The results of the present study are intriguing and should be interpreted from a clinical perspective. Dentist/hygienists should prescribe anticalculus oral rinse between the professionally cleaning of teeth. This could be a practice that would decrease the bio load in the oral cavity specially the new calculus deposits that serves as reservoir to dental plaque.

\section{REFERENCES}

1. Lederberg JA, Mccray T. Ome sweet omics: a genealogical treasury of words. Scientist 2001;15(7):8-10.

2. Paster BJ, Boches SK, Galvin JL, Ericson RE, Lau CN, Levanos VA, Sahasrabudhe A, Dewhirst FE. Bacterial diversity in human subgingival plaque. J Bacteriol 2001;183(12): 3770-3783.

3. Overman PR. Biofilm: a new view of plaque. J Contempt Dent Pract 2000;1(3):18-29.

4. Tatakis DN, Kumar PS. Etiology and pathogenesis of periodontal disease. Dent Clin North AM 2005;49(3):491-516.

5. Haffajee AD, Socransky SS. Microbial etiological agents of destructive periodontal diseases 1994; Periodontol 2000;5(1): 78-111.

6. Tagge DL, O'Leary TJ, El-Kafrawy AH. The clinical and histological response of periodontal pockets to root planing and oral hygiene. J Periodont 1975;46:527-533.

7. Lövdal A, Arno A, Schei O, Waerhaug J. Combined effect of subgingival scaling and controlled oral hygiene on the incidence of gingivitis. Acta Odontol Scand 1961;19:537-555.

8. Saini R, Saini S, Sharma S. Biofilm: a dental microbial infection. J Nat Sc Biol Med 2011;2(1):71-75.

9. Jan L. Textbook of clinical periodontology and implant dentistry. 5 ed. 748-754.

10. Van der Ouder FJG. Anti plaque agents. Rationale and prospects for prevention of gingivitis and periodontal disease. J Clin Periodont 1991;18(6):447-454.

11. White DJ. Dental calculus: Recent insights into occurrence, formation, prevention, removal and oral health effects of supragingival and subgingival deposits. Eur J Oral Sci 1997;105(5):508-522.

12. Marya CM. A text book of public health dentistry. 1st ed. New Delhi: Jaypee Brothers Medical Publishers; 2011. p. 294.

13. Darby ML, Walsh M. Dental Hygiene Theory and Practice. 4th ed. Elsevier; 2008. 